\title{
Microscopic mirrorless negative-index optical parametric oscillator
}

\author{
Alexander K. Popov, ${ }^{1, *}$ Sergey A. Myslivets, ${ }^{2}$ and Vladimir M. Shalaev ${ }^{3}$ \\ ${ }^{1}$ Department of Physics and Astronomy, University of Wisconsin-Stevens Point, \\ Stevens Point, Wisconsin 54481, USA \\ ${ }^{2}$ Institute of Physics of the Russian Academy of Sciences and Siberian Federal University, 660041 Krasnoyarsk, \\ Russia \\ ${ }^{3}$ Birck Nanotechnology Center, Purdue University, West Lafayette, Indiana 47907, USA \\ *Corresponding author: apopov@uwsp.edu
}

Received January 26, 2009; revised March 11, 2009; accepted March 13, 2009; posted March 16, 2009 (Doc. ID 106839); published April 6, 2009

The feasibility and extraordinary properties of mirrorless optical parametric oscillations in a microscopic strongly absorbing slab of negative-index metamaterial are shown. They stem from the backwardness of electromagnetic waves inherent with this type of metamaterial. (C) 2009 Optical Society of America

OCIS codes: $190.4975,190.4970,160.4236,270.1670$.

Optical negative-index (NI) metamaterials (NIMs) form a novel class of electromagnetic media that promises revolutionary breakthroughs in photonics [1]. Unlike ordinary positive-index (PI) materials (PIMs), the energy flow $\mathbf{S}$ and the wave vector $\mathbf{k}$ are counterdirected in NIMs, which determines their extraordinary linear and nonlinear optical (NLO) propagation properties. Herein, we show the feasibility of creating a microscopic, NI, backward-wave, mirrorless optical parametric oscillator (BWMOPO) that generates entangled, counterpropagating, leftand right-handed photons. Two options are explored. One is a three-wave mixing (TWM) BWMOPO that implements $\chi^{(2)}$ nonlinearities of the NIMs $[2,3]$. The other option, a four-wave mixing (FWM) BWMOPO [4], proposes independent engineering of a $\chi^{(3)}$ nonlinearity through embedded, resonant, NLO centers. In the vicinity of the resonances, $\chi^{(3)}$ is exceptionally strong and optical properties of the composite can be tailored by means of quantum control.

Figure 1(a) depicts the coupling geometry for the proposed TWM BWMOPO. The signal $h_{1}$ at $\omega_{1}$ with the wave vector $\mathbf{k}_{1}$ along the $z$ axis is a backward wave (BW); i.e., its energy flow $\mathbf{S}_{1}$ is directed against the $z$ axis. The signal enters the slab of length $L$ at $z=L$. The slab is also illuminated by a higherfrequency PI wave $h_{3}$ traveling along the $z$ axis. The two coupled waves with codirected $\mathbf{k}_{3}$ and $\mathbf{k}_{1}$ generate an idler, $h_{2}$, at $\omega_{2}=\omega_{3}-\omega_{1}$, which is also assumed to be a PI wave. The idler contributes back to the signal through TWM and thus enables optical parametric amplification (OPA) at $\omega_{1}$ through coherent energy transfer from the control field $h_{3}$ to the signal. Thus, all wave vectors are codirected along $z$, whereas $\mathbf{S}_{1}$ is counterdirected to $\mathbf{S}_{2}$ and $\mathbf{S}_{3}$. This is in strict contrast with the conventional optical parametric oscillator (OPO), where all energy flows and phase velocities are codirected, with the early proposed frequency-quasidegenerate TWM BWMOPO [5], and with the recent breakthrough realization of a nondegenerate TWM BWMOPO in a periodically poled crystal [6]. In the latter two cases, both the energy flow and the wave vector of one of the waves are opposite to all others. Assuming magnetic nonlinearity $\chi^{(2)}[2,3]$, the equations for the signal and the idler are given by

$$
\begin{aligned}
& \mathrm{d} h_{1} / \mathrm{d} z=i \sigma_{1} h_{3} h_{2}^{*} \exp [i \Delta k z]+\left(\alpha_{1} / 2\right) h_{1}, \\
& \mathrm{~d} h_{2} / \mathrm{d} z=i \sigma_{2} h_{3} h_{1}^{*} \exp [i \Delta k z]-\left(\alpha_{2} / 2\right) h_{2} .
\end{aligned}
$$

Here, $h_{j}$ are the magnetic components of the fields, $k_{j}=\left|n_{j}\right| \omega_{j} / c>0, \quad \sigma_{j}=4 \pi \chi^{(2)} \epsilon_{j} \omega_{j}^{2} / k_{j} c^{2}, \quad$ and $\Delta k=k_{3}-k_{2}$ $-k_{1} ; \alpha_{j}$ are the absorption indices. The change of $h_{3}$ along the slab is neglected, which suffices for proving the possibility of BWMOPO and for the estimate of the oscillation threshold. The depletion of the control field drops sharply outside the plasmonic resonance associated with the NI. Equation (1) exhibits three fundamental differences as compared with TWM of copropagating waves in ordinary materials; an opposite sign of $\sigma_{1}$ because of $\epsilon_{1}<0$, an opposite sign with $\alpha_{1}$ because $\mathbf{S}_{1}$ is against the $z$ axis, and the boundary conditions for $h_{1}$ to be defined at $z=L$, i.e., at the opposite edge of the slab as compared to $h_{2}$. The trans-

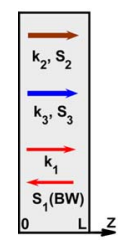

(a)
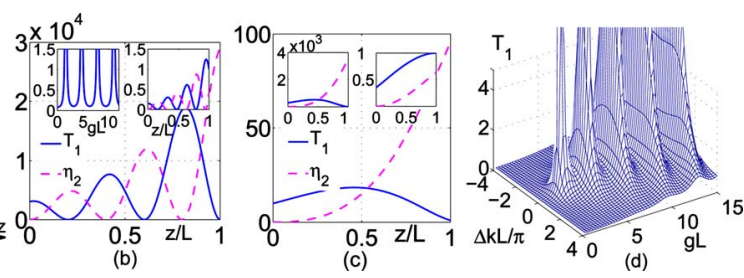

Fig. 1. (Color online) (a) Coupling geometry for a TWM BWMOPO and (b) and (c) tailored distribution of the signal, $T_{1}(z)$, and of the idler, $\eta_{2}=\left|h_{2}(z) / h_{1}(L)\right|^{2}$, along the slab at $\Delta k=0$; [(b) and main plot (c)] $\alpha_{1} L=2.5, \alpha_{2} L=-2$. (b) Main plot, $g L=7.865$; right inset, four-order decrease in $T_{1}(z)$ outside the geometrical resonance at $g L=10$; left inset, geometrical resonances in output signal $T_{10}$. (c) $g L=1.554$, left inset, $\alpha_{2} L=-\alpha_{1} L=-2.5$; right inset, $\alpha_{2} L=\alpha_{1} L=2.5$. (d) (OPO resonances) $\alpha_{2} L=3, \alpha_{1} L=2.3$. 
mission factor for the signal $T(z)=\left|h_{1}(z) / h_{1}(L)\right|^{2}$ is given by [7]

$$
T_{1}(z=0)=T_{10}=\left|\frac{\exp \left\{-\left[\left(\alpha_{1} / 2\right)-s\right] L\right\}}{\cos R L+(s / R) \sin R L}\right|^{2} .
$$

Here, $s=\left(\alpha_{1}+\alpha_{2}\right) /(4)-i(\Delta k / 2), \quad R=\sqrt{g^{2}-s^{2}}, \quad$ and $g$ $=\left(\sqrt{\omega_{1} \omega_{2}} / \sqrt[4]{\epsilon_{1} \epsilon_{2} / \mu_{1} \mu_{2}}\right)(8 \pi / c) \chi^{(2)} h_{3}$. The fundamental difference between the spatial distribution of the signal in PI and NI slabs is explicitly seen at $\alpha_{j}=\Delta k=0$. Then, Eq. (3) reduces to $T_{10}=1 /[\cos (g L)]^{2}$, which depicts a sequence of geometrical resonances as functions of the slab thickness $L$ and of the intensity of the control field $h_{3}$. This is in stark contrast with $T_{1} \propto \exp (2 g L)$ in PI media. Such an extraordinary dependence provides the feasibility of attaining the OPO threshold for the generation of the entangled, counterpropagating, left-handed $\left(\hbar \omega_{1}\right)$ and righthanded $\left(\hbar \omega_{2}\right)$ photons without a cavity at $g L$ $\rightarrow(2 j+1) \pi / 2$. A similar behavior is characteristic for distributed-feedback lasers and is equivalent to a great extension of the NLO coupling length. The crucial role of the outlined resonances is illustrated in Figs. 1(b) and 1(c). Since the idler grows toward the back facet of the slab and the signal experiences absorption in the opposite direction, the maximum of the signal for the given parameters is located closer to the back facet of the slab. The distribution of the signal along the slab and, consequently, its output value depend strongly on the difference between the absorption indices of the signal and the idler [8]. The dramatic change caused by additional amplification of the idler is readily seen from comparing the upper left and right insets of Fig. 1(c). The outcomes do not change in the case of electric nonlinearity. Assuming $\chi^{(2)} \sim 10^{-6}$ ESU, which is on the order of that for the CdGeAs 2 crystals, and $P_{3} \sim 100 \mathrm{~kW}$ focused on a spot of $D \sim 50 \mu \mathrm{m}$ one estimates that the threshold value of $g L \sim 1$ can be achieved at $L \sim 1 \mu \mathrm{m}$. It is comparable with that of the multilayer NIM samples fabricated to date [9]. Depending on the pulse duration and specific values of $\chi^{(2)}$ and $L$, the threshold intensity may appear close to the damage threshold of the film.

The basic idea of a resonant FWM BWMOPO is depicted in Figs. 2(a) and 2(b). A slab of NIM is doped by four-level nonlinear centers so that the signal frequency $\omega_{1}$ falls in the NI domain, whereas all other frequencies are in the PI domain. Here, we look for the possibility to achieve the OPO threshold for the BW signal at $\omega_{1}$ controlled by two lasers at $\omega_{3}$ and $\omega_{4}$. Owing to FWM, these three fields generate an idler at $\omega_{2}=\omega_{3}+\omega_{4}-\omega_{1}$, which may experience significant amplification that is either Raman in nature or is due to population inversion at the resonant transition $g n$ driven by the control fields. This opens an additional channel that may greatly enhance the coherent energy transfer from the control fields to the signal. The amplified idler contributes back to $\omega_{1}=\omega_{3}+\omega_{4}-\omega_{2}$ through FWM and thus causes a strongly enhanced OPA of the signal. Note that here, in contrast with far-off-resonant NLO, many orders of resonance en-

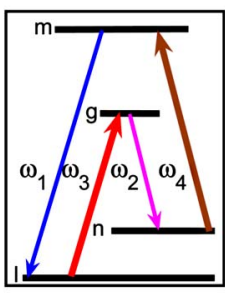

(a)

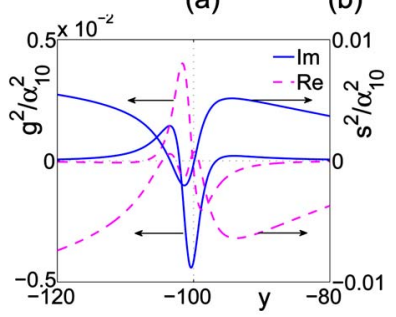

(d)

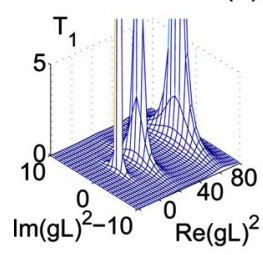

(f)

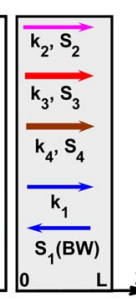

(b)

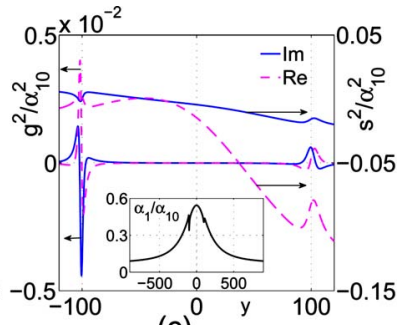

(c)

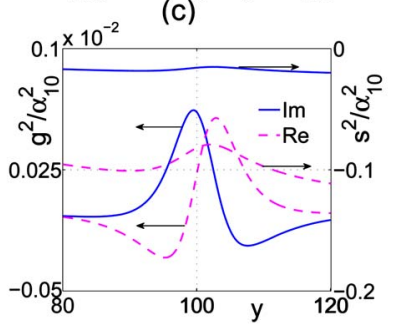

(e)

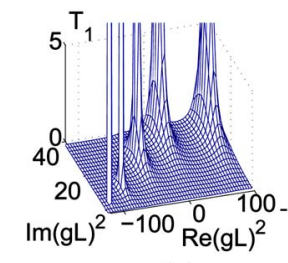

(g)

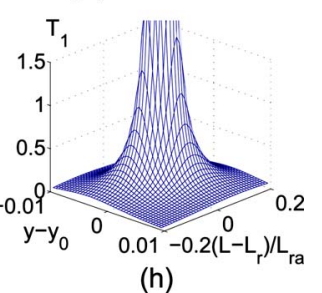

(h)
Fig. 2. (Color online) (a) Scheme and (b) coupling geometry of resonant FWM BWMOPO, (c)-(e) nonlinear interference spectral structures in $\left(g / \alpha_{10}\right)^{2}$ and $\left(s / \alpha_{10}\right)^{2}$, (f) and (g) transmittance $T_{10}$ versus $\operatorname{Re}(g L)$ and $\operatorname{Im}(g L)$, (h) BWMOPO threshold versus $\omega_{1}$ and $\alpha_{10} L . G_{3}=G_{4}=20 \mathrm{GHz}$, $\Omega_{3}=-\Omega_{4}=\Gamma_{g l}, y=\Omega_{1} / \Gamma_{l n}, y_{0}=-100.94$. (f) and (g) $\alpha_{2} L=-2$, $\alpha_{1} L=2.5$, (f) $\Delta k=0$, (g) $\Delta k L=-7 \pi$. (h) $\delta k L_{\mathrm{ra}}=0.248, L_{r} / L_{\mathrm{ra}}$ $=32.6426 . L_{\mathrm{ra}}=\alpha_{10}^{-1}$.

hancement in the NLO coupling are accompanied by strong changes in the local optical parameters driven by the control fields. This is because of modulation of the probability amplitudes and a significant population transfer. Consequently, a split and other modifications of the resonance shapes occur that stem from the quantum nonlinear interference effects (NIEs). Switching between constructive and destructive interference may serve as a tool for harnessing local optical coefficients. Alternatively, such changes can be minimized, and the majority of the amplification would come directly through the FWM coupling. Quantum interference in the vicinity of the resonances may even lead to a situation where the overall process ceases to be seen as a set of successive oneand multiphoton processes [10]. The electric amplitudes $E_{1}$ and $E_{2}$ are given by the equations

$$
\begin{aligned}
& \mathrm{d} E_{1} / \mathrm{d} z=-i \gamma_{1}^{(3)} E_{2}^{*} \exp [i \Delta k z]+\left(\alpha_{1} / 2\right) E_{1}, \\
& \mathrm{~d} E_{2} / \mathrm{d} z=i \gamma_{2}^{(3)} E_{1}^{*} \exp [i \Delta k z]-\left(\alpha_{2} / 2\right) E_{2} .
\end{aligned}
$$

Here, $\quad \gamma_{1,2}^{(3)}=\left(4 \pi\left|\mu_{1,2}\right| \omega_{1,2}^{2} / k_{1,2} c^{2}\right) \chi_{1,2}^{(3)} E_{3} E_{4}, \quad \Delta k=k_{3}+k_{4}$ $-k_{1}-k_{2}, \alpha_{j}$ are the absorption or amplification coefficients, $\omega_{2}=\omega_{3}+\omega_{4}-\omega_{1}, k_{j}=\left|n_{j}\right| \omega_{j} / c>0$, and $\chi_{1,2}^{(3)}$ are electric nonlinear susceptibilities. The signal enters the slab at the back edge at $z=L$, whereas all others are at $z=0$. Amplitudes $E_{3}$ and $E_{4}$ are assumed con- 
stant along the slab, which occurs, e.g., in the saturation regime. Equations (4) and (5) are similar to Eqs. (1) and (2). Consequently, the transmission factor $T_{1}=\left|E_{1}(z) / E_{1 L}\right|^{2}$ is given by Eq. (3), where $g^{2}$ $=\gamma_{2}^{*} \gamma_{1}$.

To demonstrate the typical threshold characteristics of the proposed FWM BWMOPO, we have carried out numerical experiments. The results are presented in Figs. 2(c)-2(h). The following model, which is characteristic of ions and some molecules embedded in a solid host, has been adopted: energy level relaxation rates $\Gamma_{n}=20, \Gamma_{g}=\Gamma_{m}=120$; partial transition probabilities $\gamma_{g l}=7, \quad \gamma_{g n}=4, \gamma_{m n}=5, \gamma_{m l}=10$ (all in $10^{6} \mathrm{~s}^{-1}$ ); homogeneous transition half-widths $\Gamma_{l g}=1$, $\Gamma_{l m}=1.9, \Gamma_{n g}=1.5, \Gamma_{n m}=1.8\left(\right.$ all in $\left.10^{12} \mathrm{~s}^{-1}\right) ; \Gamma_{g m}=5$, $\Gamma_{l n}=1$ (all in $10^{10} \mathrm{~s}^{-1}$ ); $\lambda_{2}=756$; and $\lambda_{1}=480 \mathrm{~nm}$. The density-matrix method [10] is used for calculating the intensity-dependent local parameters with accounting for the NIE in absorption, amplification, refractive indices, and in the NLO susceptibilities caused by the control fields. The changes depend on the population redistribution over the coupled levels, which in turn strongly depends on the ratio of the partial transition probabilities $\gamma_{i j}$. Figures 2(c)-2(e) depict the NIE spectral structures in the optical parameters entering into Eq. (3) at the indicated control field's strength and resonance detunings. Here, $\Omega_{1}=\omega_{1}-\omega_{m l}$; other resonance detunings $\Omega_{j}$ are defined in a similar way. Coupling Rabi frequencies are introduced as $G_{3}=E_{3} d_{l g} / 2 \hbar$ and $G_{4}=E_{4} d_{n m} / 2 \hbar$, where $d_{i j}$ are transition dipole elements. The value $L_{\mathrm{ra}}=\alpha_{10}^{-1}$ is resonance absorption length, where $\alpha_{10}$ is the fully resonant magnitude of absorption introduced by the embedded centers at $\omega_{1}=\omega_{m l}$ with all driving fields turned off. With the given parameters, the control fields cause essential changes in the level populations; $r_{l} \approx 0.487 ; \quad r_{g} \approx 0.484 ; \quad r_{n} \approx 0.014 ; \quad r_{m}$ $\approx 0.014\left(r_{l}+r_{n}+r_{g}+r_{m}=1\right)$, which is followed by an appreciable population inversion at the idle transition. A significant difference between the resonant and off-resonant NLO processes is that the NLO susceptibilities and, therefore, the parameters $\gamma_{1}$ and $\gamma_{2}$ become complex and different from each other. Hence, the factor $g^{2}$ may become negative or complex [Figs. 2(c)-2(e)]. This indicates an additional phase shift that causes further radical changes in the nonlinear propagation features, which can be tailored. Figures 2(f) and 2(g) prove that complex or negative magnitudes of $g^{2}$ may result in a situation in which phase matching $\Delta k=0$ ceases to be required and the large phase mismatch $\delta k$ introduced by the host material can be compensated through frequency resonance offsets of the fields. As discussed above and shown in Figs. 2(f) and 2(g), the output signal presents a set of distributed-feedback-type resonances. Optimization of the output signal at $z=0$ is determined by the interplay of absorption, idler gain, FWM, and the phase mismatch. Hence, the frequencies of the generated signal and idler are determined by the set of the slab's and the field's parameters with sharp resonance dependencies. One such OPO resonance predicted by Eq. (3) is shown in Fig. 2(h), which displays a narrow geometrical resonance at $L=L_{r} \approx 32.6 L_{\mathrm{ra}}$ for the optimum signal frequency offset at $\Omega_{1} \approx-101 \Gamma_{l n}$. This provides the optimum compensation for the given phase-mismatch $\delta k$ introduced by the host material, which is assumed here as $\delta k L_{\mathrm{ra}}=0.248$. Changes in $\Delta k$ introduced by the embedded centers are accounted for within the simulations. The intrinsic absorption of the host slab at $\omega_{1}$ has been assumed $90 \%$. The value of $L / L_{\mathrm{ra}}=\alpha_{10} L$ is proportional to the product of the slab length and the density of the embedded centers. Figure 2(h) indicates that the width of the oscillation resonance is less than the narrowest (here Raman) transition width and $L_{\mathrm{ra}}$. Amplification in the maxima in Fig. 2(h) reaches many orders of magnitude, which indicates the feasibility of BW cavity-less oscillations. For the given optical transitions, $G \sim 10 \mathrm{GHz}$ corresponds to the control field intensities of $P \sim 1 \mathrm{~kW}$ per spot of the diameter $D \sim 50 \mu \mathrm{m}$. Assuming a resonance absorption cross section $\sigma_{10} \sim 10^{-16} \mathrm{~cm}^{2}$, which is typical, e.g., for dye molecules and a density of the embedded centers $N \sim 10^{19} \mathrm{~cm}^{-3}$, one estimates $\alpha_{10}$ $\sim 10^{3} \mathrm{~cm}^{-1}$ and the required slab thickness to be in the microscopic scale $L \sim(1-10) \mu \mathrm{m}$. The contribution to the overall refractive index by the impurities is estimated as $\Delta n<0.5(\lambda / 4 \pi) \alpha_{10} \sim 10^{-3}$, which essentially does not change the negative refractive index.

To conclude, we propose a microscopic BWMOPO that generates entangled, counterpropagating, lefthanded and right-handed photons in an originally strongly absorbing slab of NIM. The proposal implements the backwardness of electromagnetic waves inherent to NIMs and the coherent energy transfer from the control fields to the signal through optical parametric coupling. One of the proposed schemes allows for the independent engineering of a strong NLO response and a negative refractive index in the metamaterial.

This work was supported by the U. S. Army Research Laboratory and by the U. S. Army Research Office under grants W911NF-0710261 and 50342PH-MUR.

\section{References}

1. V. M. Shalaev, Nat. Photonics 1, 41 (2007).

2. M. W. Klein, M. Wegener, N. Feth, and S. Linden, Opt. Express 15, 5238 (2007).

3. M. W. Klein, M. Wegener, N. Feth, and S. Linden, Opt. Express 16, 8055 (2008).

4. A. K. Popov, S. A. Myslivets, T. F. George, and V. M. Shalaev, Opt. Lett. 32, 3044 (2007).

5. S. E. Harris, Appl. Phys. Lett. 9, 114 (1966).

6. C. Canalias and V. Pasiskevicius, Nat. Photonics 1, 459 (2007).

7. A. K. Popov and V. M. Shalaev, Appl. Phys. B 84, 131 (2006).

8. A. K. Popov and S. A. Myslivets, Appl. Phys. Lett. 93, 191117 (2008).

9. J. Valentine, S. Zhang, T. Zentgraf, E. Ulin-Avila, D. A. Genov, G. Bartal, and X. Zhang, Nature 455, 376 (2008).

10. A. K. Popov, S. A. Myslivets, and T. F. George, Phys. Rev. A 71, 043811 (2005). 